\title{
Open peer review at four STEM journals: an observational
}

\section{overview [version 1; peer review: 1 approved, 3 approved with}

\section{reservations]}

\author{
Emily Ford (iD
}

Urban \& Public Affairs Librarian, Portland State University Library, 1875 SW Park, Portland, OR, 97207, USA

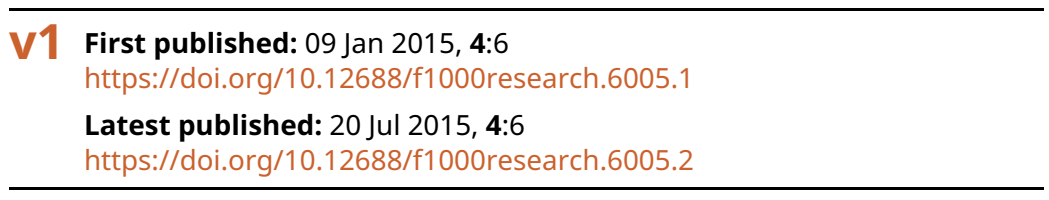

\section{Abstract}

Open peer review, peer review where authors' and reviewers' identities are disclosed to one another, is a growing trend in scholarly publishing. Through observation of four journals in STEM disciplines, PLoS One, Atmospheric Chemistry \& Physics, PeerJ, and F1000Research, an observational overview is conducted. The overview relies on defined characteristics of open peer review. Results show that despite differing open peer review implementations, each journal retains editorial involvement in scholarly publishing. Further, the analysis shows that only one of these implementations is fully transparent in its peer review and decision making process. Finally, the overview contends that journals should clearly outline peer review and editorial processes in order to allow for open peer review to be better understood and adopted by authors, reviewers, editors, and readers of science communications.

\section{Keywords}

open peer review, peer review, scholarly communication, science communication, scholarly publishing

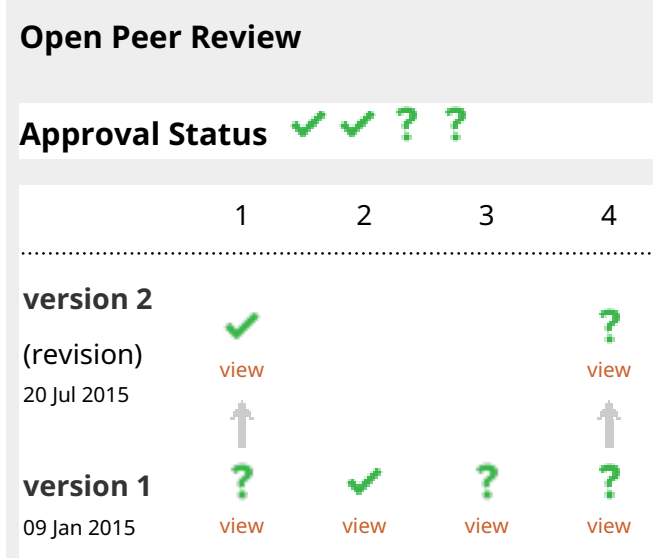

1. Peter Binfield, Peer], San Francisco, USA

2. Scott Walter, DePaul University, Chicago, USA

3. Ulrich Pöschl, Max Planck Institute for Chemistry, Mainz, Germany

4. Pandelis Perakakis (iD), University of Granada, Granada, Spain

Any reports and responses or comments on the article can be found at the end of the article. 
Corresponding author: Emily Ford (forder@pdx.edu)

Competing interests: No competing interests were disclosed. The author has no affiliation with any of the journals discussed in this article.

Grant information: The author(s) declared that no grants were involved in supporting this work.

Copyright: (c) 2015 Ford E. This is an open access article distributed under the terms of the Creative Commons Attribution License, which permits unrestricted use, distribution, and reproduction in any medium, provided the original work is properly cited. Data associated with the article are available under the terms of the Creative Commons Zero "No rights reserved" data waiver (CC0 1.0 Public domain dedication).

How to cite this article: Ford E. Open peer review at four STEM journals: an observational overview [version 1; peer review: 1 approved, 3 approved with reservations] F1000Research 2015, 4:6 https://doi.org/10.12688/f1000research.6005.1

First published: 09 Jan 2015, 4:6 https://doi.org/10.12688/f1000research.6005.1 


\section{Introduction}

In scholarly publishing open peer review (OPR) is an emerging form of peer review that incorporates disclosure of author and referee identities to one another. Although in its infancy, OPR has been adopted and implemented in a number of disciplines and their respective scholarly publications. In this article I provide some background on open peer review, addressing controversies and divergent opinions. Next I describe, examine, and discuss OPR implementations at four different science, technology, engineering, and mathematics (STEM) journals: PLoS One, Atmospheric Chemistry \& Physics, PeerJ, and F1000Research. These observations contribute to our understanding of scholarly publication and scientific communication, as we watch the evolution of scientific vetting and validity determination processes.

\section{Open Peer Review: a definition}

Unlike double-blind peer review, which is clearly defined, has clear parameters, and an arguably universal understanding by the scholarly community in how to implement it, OPR is approached and implemented in a variety of ways. There is no one universally accepted definition of OPR, which complicates investigations of its practices. As such, I rely on my previous definition, which understands OPR as any scholarly review mechanism providing disclosure of author and referee identities to one another at any point during the peer review or publication process (Ford, 2013).

Other terms used to discuss OPR are peer-to-peer review and open review. Both these phrases insinuate OPR, but some have approached is as supplementary to formal peer review processes. For example, these review implementations rely on a community's members to post comments on articles at pre-print servers, such as arXiv, or using comment features via journal websites, such as British Medical Journal and BioMed Central. It should be noted that when I mention open peer review, I discuss it as the formal process via which scholarly articles are vetted for publication.

Mentions of OPR in scholarly literature date back to Michael McGiffert's 1988 article, "Is Justice Blind? An Inquiry into Peer Review", in which McGiffert argues, based on survey results, that editors should protect the identity of authors, but that editors, "...should leave referees free to decide for themselves whether or not to make themselves known [to the author]" (p. 47, McGiffert, 1988). Over time, attitudes toward OPR have evolved, and support of OPR has grown, although it still remains debated. Although OPR is a phenomenon occurring across the academic disciplines, those in STEM are the most prolific. The first implementation of OPR occurred at Atmospheric Chemistry and Physics with its launch in 2001, which is discussed later in this article.

\section{Why Open Peer Review?}

For many, OPR addresses inherent issues in what has been the gold standard of double-blind peer review. Some see blind review processes as faulty in that referee anonymity allows for referee abuse. Others view OPR as a means to hold referees and authors accountable for their communications (Fitzpatrick, 2010; Cope \& Kalantzis, 2009; Mulligan, 2008). It has also been argued that OPR allows for easier identification of scientific misconduct (Boldt, 2011), and that over time the quality of submitted articles will improve (Hu et al., 2010; Prug, 2010). OPR affords referees the ability to gain credit for and cite their contributions to science communication (Boldt, 2011; Bornmann \& Daniel, 2010; Fitzpatrick, 2010; Prug, 2010; Pöschl, 2009). More broadly speaking, OPR provides the scholarly community an insight into author/referee conversations during the review process. Surfacing these conversations provides readers an expanded contextual discussion of the subject at hand, and enriches science communication for all stakeholders (Lipworth \& Kerridge, 2011; Fitzpatrick, 2010; Friedman et al., 2010; Maharg \& Duncan, 2007). Finally, perhaps the most convincing pro argument for OPR asserts that OPR processes allow for quicker publication and dissemination of scientific findings (Hu et al., 2010; Cope \& Kalantzis, 2009; Pöschl, 2004).

One of the major arguments against open peer review is the perceived protection afforded both authors and reviewers in a blind process. For junior researchers serving as reviewers, blind review may allow them to feel more able to provide honest constructive criticism to senior researchers. Similarly, as authors, blind review is perceived as protecting junior researchers from public humiliation (Godlee, 2003). It has also been noted that some reviewers refuse to participate in OPR implementations, or still have concerns about them (Janowicz \& Hitzler, 2012). These concerns still pervade conversations about OPR. Most recently, a survey of $B M C$ Pharmacology and Toxicology Editorial Board Members surfaced continuing concerns regarding OPR at the journal. Despite these concerns BMC Pharmacology and Toxicology decided to "...continue with open peer review at BMC Pharmacology and Toxicology because of the ethical grounds for doing so and because the potential benefits outweigh the negatives", (p. 4, Moylan et al., 2014). This is evidence that despite continuing concerns and resistance to it, OPR will continue to be implemented and evolve in STEM publishing. As such, scholars should understand OPR implementations in order to further innovate and evolve scholarly publishing and scientific communication.

\section{Methods}

Four STEM journals claiming to use open peer review processes were chosen for observation. These four journals were selected because they represent a difference in relative age, their perceived stature or authority in STEM, and for the salience of information regarding OPR on their respective websites. To review and understand the four different peer review implementations, this observation relied on the eight OPR characteristics I identified in 2013. I relied on these characteristics because there is no other documented common vocabulary used to discuss and analyze open peer review. The characteristics are cited in full below:

- $\quad$ Signed review refers to submitted reviews signed by the referee that are either published alongside articles at the time of publication or are signed when an author receives them.

- $\quad$ Disclosed review refers to a process in which referees and authors know each others' identities during the peer review process, enabling them to engage in discussion or discourse.

- Editor-mediated review is a characteristic found in most open peer review processes. Editor mediation is any work done by a journal editor to facilitate open peer review. 
This may include editorial preselection of articles and/or final decision-making for acceptance or rejection of articles. The editor-mediated portion of any open peer review process may or may not be publicly disclosed.

- Transparent review refers to complete openness to a distinct community or the public. It allows a public community to watch peer review unfold. Authors and the public know referees' identities, and referees know authors' identities. Author responses to referee comments are public. In transparent review the public can see manuscripts, reviews, and replies from authors and public reviewers as well as the published articles.

- Crowd-sourced review is a public review process in which any community member may contribute to the article review. In crowd-sourced review there is no limit to the number of comments or reviews an article may receive. In some proposed implementations of crowdsourced review, there is little editorial mediation of article reviews. Rather, authors may simply submit papers to a preprint server or other community for crowd-sourced commentary.

- Pre-publication review occurs prior to article publication, and typically occurs in a public space such as a pre-print server.

- Synchronous review occurs at the same time as publication of the article. In the literature, synchronous review is approached only theoretically, as part of a novel and completely iterative publishing model.

- Post-publication review occurs after an article is published, much like commentary on a blog post (pp. 314-315, Ford, 2013).

Using these characteristics I examined information for authors and about each publication at their respective websites, promotional materials, blogs, and other materials discussing the OPR processes at each journal. Data for these observations was gathered in mid to late 2013. Publisher/journal policies and practices may have since changed.

\section{The Journals \\ PLoS One}

PLoS One is an international publication of Public Library of Science, a not-for-profit publisher and open access advocacy organization. The journal was formed around the philosophy and practice that all research using scientifically sound research methods should be published regardless of its results, novelty, and/or impact. The journal publishes research articles from science and medical disciplines, including those reporting negative results. By publishing research from multiple disciplines, the journal boasts "PLoS One facilitates the discovery of the connections between papers whether within or between disciplines".

PLoS One launched in December 2006 and has since seen tremendous growth. It is indexed in numerous databases; is frequently cited as a source of research in news and popular media; and has received positive press for its review process. Even John Bohannon, a science journalist who undertook a sting operation of open access journals in an attempt to uncover poor publishing practices, acknowledged the strength of PLoS One's review process (Bohannon, 2013, ๆ 9).

All articles published in PLoS One carry Creative Commons attribution licenses. Although most authors publishing in the journal pay article processing charges (APCs), the journal makes exceptions and waives publishing fees for unfunded research. Moreover, the APC fee model at PLoS One takes into account an author's country of origin, and whether it is a high, lower middle, or low income nation. In this way the publication aims to make more viable open access publication for authors with disparate economic means.

Compared to the other journals I discuss in this article, PLoS One conservatively approaches OPR. The journal's peer review process only exhibits a few OPR characteristics, and even then these characteristics are not consistently implemented. It does, however, always use a form of editor-mediation for reviewing and publishing its content. Each submitted article is assigned an Academic Editor, who then determines whether submissions should be considered for peer review, and who then facilitates the peer review process. According to the journal's review guidelines, these Academic Editors may, "...conduct the peer review themselves, based on their own knowledge and experience; they can take further advice through discussion with other members of the editorial board; they can solicit reports from further referees".

While the editorial mediation of journal articles at PLoS One always occurs, other characteristics of OPR do not. Signed reviews are optional, but they are strongly encouraged. According to its peer review guidelines, "If Peer Reviewers are willing, then they are also identified to the author at the time of decision." PLoS One does not post reviewer comments to the web alongside published articles, so readers do not benefit from reading discussions that occurred about the topic prior to publication. In addition to PLoS One's version of signed review, the journal enables crowd-sourced review, which occurs post-publication. Readers may comment on articles that have been published. One unique aspect of this crowdsourced review process is that PLoS One surfaces any media coverage of published articles by linking to them in an article's comments section. The result is that the journal is able to create a record of the impact and conversation an article elicits outside of the PLoS One platform and community.

\section{Atmospheric Chemistry and Physics}

Perhaps the oldest of the open peer reviewed publications is Atmospheric Chemistry and Physics (ACP), the European Geosciences Union's journal. The journal, which launched in September of 2001 (Pöschl, 2004), publishes research articles, review articles, technical notes, peer-reviewed comments, correigenda, and supplementary materials. All published content is published under a creative commons 3.0 attribution license and authors are subject to APCs.

Following an article's submission to $A C P$ and brief editorial review, it is then hosted on the journal's pre-print server, Atmospheric Chemistry and Physics Discussion Papers (ACPD) for peer review and crowd-sourced discussion. On this platform reviewer comments and crowd-sourced comments are publicly available. After the discussion period for a paper ends, the ability to comment on the paper 
is turned off, and the journal editor makes final publishing decisions using submitted referee and public reviews. When the editor accepts an article for publication, the article is published at $A C P$, where it will also link to its pre-print version, and referee and public comments at $A C P D$. ACP does not host public commentary on published articles. Although $A C P$ views its peer review process as completely transparent (Pöschl, 2004), it is not. Reviewers may choose to disclose their identities, or they may choose to remain anonymous. True transparency of the review process can only occur when reviewers and public commenters have disclosed their identities.

In addition to editor mediation and crowd-sourced reviewing, $A C P$ 's process employs disclosed review and pre-publication review. It could be argued that synchronous review occurs, yet $A C P$ does not consider papers posted at $A C P D$ to be "published." Because these papers are not considered published, their review is not synchronous.

\section{PeerJ}

PeerJ is an individual membership-based publisher in the biological and medical sciences. All works published by PeerJ are licensed with a Creative Commons Attribution 3.0 Unported license. Its main publication, PeerJ only publishes research articles. All other publication types are referred to PeerJ PrePrints, its pre-print repository and publication. PeerJ is young. The publisher first announced its publication model in June 2012, and published its first article on February 13, 2013. Its model relies on membership, where individuals pay one fee to PeerJ and become lifetime members. Based on an individual's membership level-basic, enhanced, or investigator-individuals may publish in PeerJ a dedicated number of times per year.

The publishing model at PeerJ is similar to the pre-print/publication relationship between $A C P$ and $A C P D$ in that it maintains two publishing platforms: PeerJ and PeerJ PrePrints . Arguably, PeerJ PrePrints is not a publication, but a pre-print repository service. However, unlike ACP, PeerJ considers PeerJ PrePrints a publication, so I will treat it as such alongside PeerJ.

Only paid PeerJ members may publish work in PeerJ. Works may first be submitted to PeerJ PrePrints, or may be directly submitted to PeerJ. PeerJ's article review, acceptance and publication model mirrors PLoS One's; PeerJ accepts scientifically sound research and does not consider an article's "novelty, interest, or impact" as part of its editorial criteria. Prior to review, submissions to PeerJ undergo editorial vetting by assigned Academic Editors. These Academic Editors are responsible for facilitating peer review of assigned articles to be completed by at least one reviewer, and making final publication decisions. Additionally, Academic Editors are attributed alongside each published article. Authors and reviewers alike are "encouraged" to post the full peer review cycle online alongside final versions of articles, but the journal does not mandate it. Based on information from PeerJ's website, it is unclear whether author and reviewer identities are disclosed to one another during the review process.

Unlike the crowd-sourced review occurring at PLoS One and $A C P$, PeerJ utilizes a broader model named Q\&A. PeerJ's Q\&A incorporates not only questions and answers regarding pre-prints and articles-which can be posed at the paragraph and figure level-it also allows for free-standing questions. In this way, Q\&A is intended to be a platform for anyone to participate in scientific conversation. At PeerJ Q\&A includes an incentive system for individual participants in the community. Contributing individuals are awarded points for their engagement. For example, one earns 100 points for authoring an article, 35 points for contributing an open review, etc. These points are displayed on members' profile pages.

PeerJ has future plans to expand PeerJ PrePrints. The publisher hopes to allow authors to share as much or as little of their publications as they wish. They may decide to openly publish a title, title and abstract, or the whole paper. Additionally, PeerJ PrePrints will allow for authors to share papers "privately" with only particular users, only the PeerJ community, or fully open on the web.

\section{F1000Research}

Finally, the fourth of the journals I discuss, F1000Research, most consistently exhibits OPR characteristics. It is an open access journal published by Faculty of 1000 . The publisher calls it one of “... four unique services that support and inform the work of life scientists and clinicians" provided by the publisher. F1000Research published its first approved article in July 2012, only six months after Faculty of 1000 announced the new publication (Lawrence, 2012a, 2012b). For published data the journal utilizes a Creative Commons No Rights Reserved license; it requests attribution for works, but anyone anywhere is free to use, build upon, and manipulate works. For published articles the journal uses a Creative Commons Attribution license. The journal itself includes case reports, clinical practice articles, commentary, correspondence, data articles, method articles, opinion articles, research articles, reviews, short research articles, study protocols, systematic reviews, thought experiments, and web tools in the Life Sciences. Authors submitting articles to F1000Research pay APCs (F1000Research, 2013a).

At F1000Research articles undergo a peer review process after they are published. As such, F1000Research is the only publication I discuss that uses a post-publication review process. In this way the journal is able to speed up publication timelines to disseminate scholarly work; the journal publishes articles within one week of submission. As stated in its referee guidelines, the journal publishes submitted articles that pass initial editorial review for "content, quality, tone and format" as well as completeness, plagiarism, ethical standards, and adherence to author guidelines. In addition to reviews provided each work by two or three designated expert referees-which are attributed to the reviewer and are published online with the work - the scientific public (those affiliated with scientific or medical organizations) may comment on any published article. Any author responses to referees are also public.

In this publication model it is possible for articles to receive unanimous negative reviews. In this case, articles remain "published", but are removed from the site's default search. The site's interface clearly delineates a work's referee status and comments using icons to distinguish its status: approved with reservations, approved, and not approved. It is important to note that F1000Research does not 
consider these statuses as equivalent to the accepted, accepted with revisions, and rejected statuses that one sees in closed review processes. Rather, as stated in the FAQs:

The term Approved means that the referee thinks that the article is good and has either no suggested revisions or only minor revisions. The term Approved with Reservations means that the referee agrees that the article has scientific merit and is fundamentally sound but would like the author to make further changes to the manuscript. This is approximately equivalent to a request for major revisions or several minor revisions in a traditional journal. In every case, even when all referees approve of the article, future versions are welcome.

Because F1000Research publishes and attributes all referee responses and author comments, it adheres to a fully transparent peer review process. In addition to transparent reviews provided by pre-selected referees, crowd-sourced review occurs when individuals comment on published articles. The journal exhibits most other OPR characteristics; its reviews are editor-mediated, transparent, referee and author identities are disclosed, and reviews are signed. The only OPR characteristics not exhibited by F1000Research's process are those related to review timing. Since all review at F1000Research occurs post-publication, the journal does not exhibit pre-publication and synchronous review characteristics. Once a work has been vetted by editors and is made public on the journal's site, the journal considers it published. Because review occurs post publication, authors receiving critical feedback are encouraged to revise and submit updated versions of articles that will, again, be refereed. The journal uses CrossRef's CrossMark identification service to assist readers in tracking these article versions and relationships. Even if an author publishes an updated article, previous versions remain published. In this way, publication at F1000Research is a good example of the iterative process of publishing and scientific knowledge and conversation.

\section{Discussion}

I examined four journals that boast open peer review processes, PLoS One, ACP, PeerJ, and F1000Research. None of these journals have implemented OPR in the same manner, but they do exhibit many of the same OPR characteristics. Each journal exhibits a form of editor-mediation and each journal vets submitted articles prior to publication for basic quality, scope and adherence to author guidelines. While for some journals, such as F1000Research, this is as far as an editor's work goes before an article is published, others, such as $A C P$ and PeerJ, allow editors to make final publishing decisions.

Each of the journals allow for some form of crowd-sourced review. At PeerJ the Q\&A section includes commentary on articles; it also includes other avenues for the public to engage in scientific conversations. At PLoS One, however, commentary on articles occurs only after an article has been published, and also includes links to all media coverage of articles. Unlike PLoS One, PeerJ, and F1000Research's crowd-sourcing implementations, $A C P$ 's process only allows for crowd-sourced commentary prior to publication articles during the discussion phase of the OPR process.

Another commonality between these publications is their varying allowance, encouragement, or mandate for reviewers to sign their commentary. Referees may choose to remain anonymous at $A C P$ and PeerJ (even though disclosure is strongly encouraged), whereas F1000Resarch and PLoS One require referees to disclose their identities. Since author/reviewer identity disclosure is a defining factor of OPR, it could be argued that those articles where reviewer commentary is not attributed are not truly open peer-reviewed. The motivation for publishers to encourage rather than require openness most likely stems from their desire to encourage more authors and reviewers to participate in alternative peer review processes. Publishers may also be attempting to be mindful of different discipline's accepted publishing practices. In my own view, by not mandating public attributed review, publishers are weakening the power of OPR. However, incremental steps in OPR implementation are necessary to encourage participation and to move OPR to a completely transparent standard in the future.

Two of the journals discussed above include article pre-print mechanisms. Pre-print servers and mechanisms introduce confusion into understanding publishing and open peer review. Just when is something that is open to be read on the web considered "published?" Where $A C P$ does not consider papers posted to its pre-print space, $A C P D$, as "published", PeerJ PrePrints is considered by its publisher a publication. Defining a moment of when scholarly work is "published" will continue to evolve as does scholarly publishing and open peer review processes.

Of the four publishers discussed, I maintain that F1000Research exhibits what we should consider the gold standard of transparent and OPR processes. The publication's process is completely transparent; it publishes all commentary with attribution and makes salient referee decisions. Moreover, the mechanism it uses to track and correlate article versions and updates enhances and opens scholarly conversations. Yet, F1000Research maintains its editorial voice via editor-mediation prior to an article's publication and by suppressing from search results articles receiving unanimous negative reviews.

\section{Conclusion}

Scholarly journals are beginning to challenge traditional peer review practices by implementing OPR, yet each OPR implementation differs. By observing four different implementations of OPR I conclude that few OPR journals implement truly transparent review, yet each implementation values editorial work. Further, I maintain that distinguishing between publicly available preprints and publicly available published articles unnecessarily muddies the waters in understanding OPR. As OPR implementations proliferate, it is pertinent for journals to clearly outline any peer review process so that readers, authors, and reviewers can fully understand peer review implementations, decision making processes, and to provide for editorial transparency.

\section{Competing interests}

No competing interests were disclosed. The author has no affiliation with any of the journals discussed in this article.

\section{Grant information}

The author(s) declared that no grants were involved in supporting this work. 
Bohannon J: Who's afraid of peer review? Science. 2013; 342(6154): 60-65. PubMed Abstract | Publisher Full Text

Boldt A: Extending ArXiv.org to Achieve Open Peer Review and Publishing. Journal of Scholarly Publishing. 2011; 42(2): 238-242.

Publisher Full Text

Bornmann L, Daniel HD: Reliability of reviewers' ratings when using public peer review: a case study. Learn Publ. 2010; 23(2): 124-131.

Publisher Full Text

Cope W, Kalantzis M: Signs of epistemic disruption: Transformations in the knowledge system of the academic journal. First Monday. 2009; 14(4-6). Publisher Full Text

Fitzpatrick K: Peer-to-peer Review and the Future of Scholarly Authority. Social Epistemology. 2010; 24(3): 161-179.

Publisher Full Text

Ford E: Defining and Characterizing Open Peer Review: A Review of the Literature. Journal of Scholarly Publishing. 2013; 44(4): 311-326.

Publisher Full Text

Friedman R, Whitworth B, Brownstein M: Realizing the Power of Extelligence: A New Business Model for Academic Publishing. International Journal of Technology, Knowledge \& Society. 2010; 6(2): 105-117.

Reference Source

Godlee F: Peer Review in Health Sciences (2nd ed.). London: BMJ Books. 2003. Reference Source

Hu C, Zhang Y, Chen G: Exploring a New Model for Preprint Server: A Case Study of CSPO. Journal of Academic Librarianship. 2010; 36(3): 257-262. Publisher Full Text

Janowicz K, Hitzler P: Open and transparent: the review process of the
Semantic Web journal. Learn Publ. 2012; 25(1): 48-55.

Publisher Full Text

Lipworth W, Kerridge IH, Carter SM, et al:: Should Biomedical Publishing Be "Opened Up"? Toward a Values-Based Peer-Review Process. J Bioeth Inq. 2011 8(3): 267-280

Publisher Full Text

Maharg P, Duncan N: Black Box Pandora's Box or Virtual Toolbox? An

Experiment in a Journal's Transparent Peer Review on the Web. International

Review of Law Computers \& Technology. 2007; 21(2): 109-128.

Publisher Full Text

McGiffert M: Is Justice Blind? An Inquiry into Peer Review. Scholarly Publishing. 1988; 20(1): 43-48.

Moylan EC, Harold S, O'Neill C, et al.: Open, single-blind, double-blind: which peer review process do you prefer? BMC Pharmacol Toxicol. 2014; 15(1): 55 Publisher Full Text

Mulligan A: Quality, certification and peer review. Information Services \& Use. 2008; 28(3-4): pp. 197-214.

Reference Source

Pöschl U: Interactive journal concept for improved scientific publishing and quality assurance. Learn Publ. 2004; 17(2): 105-113.

Publisher Full Text

Pöschl U: Interactive Open Access Peer Review: The Atmospheric Chemistry and Physics Model. Against the Grain. 2009; 21(3): 26-32.

Reference Source

Prug T: Open-process academic publishing. Ephemera: Theory \& Politics in Organization. 2010; 10(1): 40-63.

Reference Source 


\section{Open Peer Review}

\section{Current Peer Review Status: ? $\checkmark$ ?}

\section{Version 1}

Reviewer Report 04 March 2015

https://doi.org/10.5256/f1000research.6426.r7267

(C) 2015 Perakakis P. This is an open access peer review report distributed under the terms of the Creative Commons Attribution License, which permits unrestricted use, distribution, and reproduction in any medium, provided the original work is properly cited.

\section{Pandelis Perakakis \\ Department of Psychology, University of Granada, Granada, Spain}

Thank you for this interesting observation article and for the invitation to contribute my open peer review.

In the beginning, the article tries to disambiguate different "flavours" or implementations of open peer review (OPR) by identifying a number of OPR characteristics. Regarding this effort I would like to note the following issues that I think should be somehow addressed in a future version of the article:

As already noted by Peter Binfield, the definition offered is limited to only one of the identified OPR characteristics, namely whether the reviews are signed or not.

It is not clear what is the difference between reviews "signed when the author receives them" and disclosed reviews. It seems that in both cases the names of authors and reviewers are disclosed from the beginning of the review process.

Is disclosure of the reviewer's identity necessary to initiate a discussion between authors and reviewers? Perhaps what you refer to here is an infrastructure to allow multiple iterations during the review process, as provided for example by Frontiers. This is different from simply revealing the name of the reviewer.

There is a clear distinction between an unsolicited comment on an article and a formal review process by expert peers that receive a review invitation either by an experienced editor or the authors themselves.

What is the alternative to editor mediation in peer review, if we consider that unsolicited commentaries do not constitute formal peer reviews? Is there another model where formal peer review is not editor-mediated? I know we proposed such model that we called authorguided peer review in Perakakis, P., Taylor, M., Mazza, M., Trachana, V. (2010). Natural selection of academic papers. Scientometrics, 85 (2), pp. 553-559, but I am not aware of any implementations. I repeat that I do not think we should consider crowd-sourced comments 
as equal to formal peer reviews.

And some other comments to consider:

PLOS ONE clearly does not qualify as an OPR journal by any standards. Instead, I would be more interested to see a discussion on the implementation of OPR by Frontiers.

I suspect there is a misunderstanding around the terms double-blind and single-blind review. The standard in most journals and disciplines is single-blind review where authors are blind to the name of the reviewer, but reviewers know the name of the authors. Doubleblind refers to the process where neither authors nor reviewers are aware about the identity of each other.

It is not entirely correct that PLOS ONE "waives publishing fees for unfunded research". What they do is ask for proofs that authors cannot cover the APCs even from their own budget, sometimes even asking for personal financial records, which by the way I find unacceptable. Proving that there is no formal funding to publish the particular research is not enough to waive APCs.

I definitely agree that a comparison table is needed.

I detected that the references to the articles by Lawrence are missing. Please double check that all references are included.

Thank you again for the opportunity to participate in this process that is very close to my ideal model of OPR and I will be looking forward to a revised version of your manuscript.

Competing Interests: No competing interests were disclosed.

I confirm that I have read this submission and believe that I have an appropriate level of expertise to confirm that it is of an acceptable scientific standard, however I have significant reservations, as outlined above.

Reviewer Report 03 March 2015

https://doi.org/10.5256/f1000research.6426.r7273

(C) 2015 Pöschl U. This is an open access peer review report distributed under the terms of the Creative Commons Attribution License, which permits unrestricted use, distribution, and reproduction in any medium, provided the original work is properly cited.

\section{Ulrich Pöschl}

Multiphase Chemistry Department, Max Planck Institute for Chemistry, Mainz, Germany

Thanks to the author for an interesting perspective article on open peer review. Following an invitation to review this article, I would like to request a couple of specific corrections and add a general comment and suggestion.

1. Correction of Erroneous Statement: "Although ACP views its peer review process as completely transparent (Pöschl, 2004), it is not." 
Neither the cited reference nor the ACP journal web pages claim that the peer review process of ACP would be "completely transparent". There are good reasons to maintain an option for referees to remain anonymous, and this is also very clearly specified on the journal web pages as well as in the referenced article and in a more comprehensive review article (Pöschl, 2012 as referenced and linked below). Thus, it would indeed be wrong to claim "complete transparency" for the open peer review process for ACP, but it is also wrong to insinuate that ACP or the cited article would have raised this claim. Please discard or correct the erroneous statement accordingly. Moreover, please complement or replace the reference to Pöschl 2004 by a reference to Pöschl 2012, because the latter is more comprehensive and up-to.date:

Pöschl U (2012) Multi-stage open peer review: scientific evaluation integrating the strengths of traditional peer review with the virtues of transparency and self-regulation. Front. Comput. Neurosci. 6:33. doi: 10.3389/fncom.2012.00033 http://journal.frontiersin.org/article/10.3389/fncom.2012.00033/abstract

2. Correction of Erroneous Statement: "It could be argued that synchronous review occurs, yet $A C P$ does not consider papers posted at ACPD to be "published." Because these papers are not considered published, their review is not synchronous."

Neither the ACP journal web pages nor the articles providing an authoritative description of the ACP open peer review (Pöschl, 2004 \& 2012) have ever indicated that the papers posted in the ACP discussion forum, ACPD, would not be "published". Well on the contrary, all relevant journal web pages emphasize explicitly that the discussion papers posted and reviewed/discussed in ACPD are publications with permanent public accessibility, archiving and citability. Also my articles explaining the concepts of interactive open access publishing and multi-stage open peer review (Pöschl, 2004 and 2014) specify and emphasize that the discussion papers published in ACPD - as well as in the fifteen interactive open access sister journals of the European Geosciences Union (EGU) - are indeed publications. Thus, it is inappropriate to insinuate the opposite. Please discard or correct the erroneous statement accordingly.

http://www.egu.eu/about/statements/position-statement-on-the-status-of-discussionpapers-published-in-egu-interactive-open-access-journals/

http://www.atmospheric-chemistry-and-

physics.net/general_information/publication_policy.html

http://www.atmospheric-chemistry-and-

physics.net/review/review_process_and_interactive_public_discussion.html http://www.atmospheric-chemistry-and-physics.net/general_information/faq.html http://www.ingentaconnect.com/content/alpsp/lp/2004/00000017/00000002/art00005 http://journal.frontiersin.org/article/10.3389/fncom.2012.00033/abstract

\section{General Comment and Suggestion:}

I understand and respect that the author prefers a fully transparent form of open peer review as implemented by F1000Research. However, F1000Research is a relatively recent follow-up on a series of earlier initiatives that have been pursuing open peer review and are reaching back into the last century. For example, see the British Medical Journal (BMJ), the Electronic Transactions on Artificial Intelligence (ETAI), the Journal of Interactive Media in 
Education (IIME) and other journals with open peer review as referenced in the recent "research topic" (special issue) on open evaluation (including open peer review) in the open access journal Frontiers in Computational Neuroscience:

\section{http://journal.frontiersin.org/researchtopic/137}

As detailed in my contribution to that collection of articles (Pöschl 2012) and confirmed by independent studies referenced/linked below, ACP and its sister journals are by most standards of scientific publishing more successful and more efficient than comparable journals with traditional or alternative forms of peer review:

http://www.atmospheric-chemistry-and-

physics.net/pr_acp_is_interactive_open_access_publishing_able_to_identify_high_impact_submissions.pdt

\section{http://www.biomedcentral.com/1471-2288/13/74}

Overall, I see no well-founded basis for the claim that the particular form open peer review practiced by F1000Research would deserve the attribute "gold standard of open peer review", and I would suggest to substantiate or drop this postulate.

Competing Interests: I am the initiator and chief executive editor of the interactive open access journal Atmospheric Chemistry and Physics (ACP), which is one of the journals discussed in this article, and I have chaired the publications committee of the European Geosciences Union (EGU) with fifteen sister journals pursuing the same approach of open peer review.

\section{I confirm that I have read this submission and believe that I have an appropriate level of expertise to confirm that it is of an acceptable scientific standard, however I have significant reservations, as outlined above.}

Reviewer Report 12 January 2015

https://doi.org/10.5256/f1000research.6426.r7270

(C) 2015 Walter S. This is an open access peer review report distributed under the terms of the Creative Commons Attribution License, which permits unrestricted use, distribution, and reproduction in any medium, provided the original work is properly cited.

\section{Scott Walter}

John T. Richardson Library, DePaul University, Chicago, IL, USA

The study applies the tools of content analysis to the stated editorial policies and procedures of a small number of journals in STEM disciplines adhering (to a greater or lesser degree) to the principles of open peer review. To promote replicability of the study and/or comparison between the set of journals included in the current study and other sets of journals, the study might be revised to include a table documenting the degree to which each journal under consideration did or did not meet the stated OPR principles. While the study is limited to review of relatively new 
publications and publication models in a defined field, it might also be revised to suggest future research opportunities, e.g., the degree to which OPR principles are employed in more established journals, the degree to which OPR experiments are being explored in other fields.

Competing Interests: No competing interests were disclosed.

\section{I confirm that I have read this submission and believe that I have an appropriate level of expertise to confirm that it is of an acceptable scientific standard.}

Author Response 25 Jan 2015

Emily Ford, Portland State University Library, 1875 SW Park, Portland, USA

Thank you for your comments and feedback. The suggestion of a table, I think, will help to clearly communication these observations, so a revision will include one. One of the things that I have struggled with is how to represent what might be happening in other disciplinary communities. From what I have found, OPR seems to have started in STEM disciplines. I'm finding it difficult to wrap my thoughts and ideas around OPR in the humanities since humanities publishing is also changing with new ways to express scholarship, aka the digital humanities. In this way it is easier (for me) to begin observing and evaluating OPR in STEM publications.

Again, thank you for your thoughts and comments, and I look forward to more comments after I re-submit this article.

Competing Interests: No competing interests were disclosed.

Reviewer Report 12 January 2015

https://doi.org/10.5256/f1000research.6426.r7269

(C) 2015 Binfield P. This is an open access peer review report distributed under the terms of the Creative Commons Attribution License, which permits unrestricted use, distribution, and reproduction in any medium, provided the original work is properly cited.

\section{Peter Binfield}

Peer], San Francisco, CA, USA

Thank you for an interesting article which overviews an emerging form of publication / peer review

\section{General Comments:}

At an early point, the author defines OPR as simply naming the authors and reviewers ("any scholarly review mechanism providing disclosure of author and referee identities to one another at any point during the peer review or publication process"), but then goes on (e.g. in the $2^{\text {nd }}$ half of para 1 
of "Open Peer Review") to discuss it as if it also includes making those comments publicly available at the time of publication (something which is certainly considered part of OPR by many people). Therefore, the author either needs to expand their definition, or they need to word their commentary differently.

For example, when the author makes statements that:

"More broadly speaking, OPR provides the scholarly community an insight into author/referee conversations during the review process."

They are implying that the community can read the comments upon publication. This is not the case when using the narrow definition which the author has adopted (which is stated as "any scholarly review mechanism providing disclosure of author and referee identities to one another at any point during the peer review or publication process" and thus does not explicitly include the possibility that peer-review comments will be made public upon publication).

I suggest that the author clearly defines what they mean as OPR for the purposes of this article

Note: It is interesting that all the studied journals are OA - is that worth a discussion? Is OA more naturally favorable to OPR? Are there any subscription journals operating OPR? If not, why not?

\section{Edits:}

"have approached is as supplementary" should read "have approached it as supplementary"

PLoS One should be written PLOS ONE throughout

"individuals may publish in PeerJ a dedicated number of times per year" edit to: "individuals may publish in PeerJ a specified number of times per year"

"peer review of assigned articles to be completed by at least one reviewer," change to: "peer review of assigned articles to be completed by at least two reviewers,"

Grammar needs fixing in: "In addition to reviews provided each work by two or three designated expert referees"

\section{Notes:}

- "By publishing research from multiple disciplines, the journal boasts "PLoS One" use of the word 'boasts' seems unnecessarily emotional here.

"The first implementation of OPR occurred at Atmospheric Chemistry and Physics with its launch in 2001" This seems unlikely. Is there a reference? The article then says: "Perhaps the oldest of the open peer reviewed publications is Atmospheric Chemistry and Physics". So which is it? The oldest? Or 'perhaps' the oldest?

"In addition to PLoS One's version of signed review, the journal enables crowd-sourced review, which occurs post-publication." Although I note the authors definition of "crowd sourced review" (their bullet 5 in Methods), it is worth saying that at the time this article was written, PLOS ONE did not (and still doesn't, I believe) regard (or promote) their commenting facility as a form of review. Instead they regard it as a way to enable "post publication commentary". Same goes for Peer]. 
"One unique aspect of this crowd-sourced review process is that PLOS One surfaces any media coverage of published articles by linking to them" This isn't necessarily unique, Peer] does this as well via the ability for any user to "add link". Also, many other journals do this via their article level metrics provision.

"Peer] is an individual membership-based publisher in the biological and medical sciences." At the time this article was written this was correct. However, since that time, the journal changed the description of its model to "publication plans" rather than "memberships"

"All works published by PeerJ are licensed with a Creative Commons Attribution 3.0 Unported license" Correct at the time the article was written. However, since that time the journal has upgraded to CC BY 4.0

"Based on information from Peerf's website, it is unclear whether author and reviewer identities are disclosed to one another during the review process." I can clarify this - reviewers are encouraged to name themselves ( $40 \%$ choose to do so). If they do so, then the authors are first made aware of their identities in the decision letter that includes the comments of that reviewer.

"I examined four journals that boast open peer review processes, PLoS One,". Actually, I am not sure that PLOS ONE would claim this fact (and the author themselves observes that very few elements of OPR are provided by PLOS ONE)

"whereas F1000Resarch and PLOS One require referees to disclose their identities". This is not the case for PLOS ONE (unless something has changed which I am not aware of). The default for PLOS ONE reviewers is to remain anonymous.

Competing Interests: I am the Publisher of Peer] and used to be the Publisher of PLOS ONE, both surveyed in this report.

\section{I confirm that I have read this submission and believe that I have an appropriate level of expertise to confirm that it is of an acceptable scientific standard, however I have significant reservations, as outlined above.}

Author Response 25 Jan 2015

Emily Ford, Portland State University Library, 1875 SW Park, Portland, USA

Thank you for your thoughtful comments. Your working knowledge of both PeerJ and PLOS ONE will benefit this article's revision, and I appreciate you offering this knowledge in your review.

Regarding your note about OPR and the overlap with OA journals: yes, OA journals are generally more sympathetic and willing to try OPR. Since you ask it is clear to me that I have not adequately discussed this overlap in the article, which I will be sure to in a revision.

Thank you again for your comments. 
Competing Interests: No competing interests were disclosed.

\section{Comments on this article}

\section{Version 1}

Reader Comment 05 Mar 2015

Jason Moore, Cleveland State University, USA

I'm not sure if this should be in the scope of this paper, but at some point in history many academic journals had open peer review. I often find the correspondence between author and reviewers appended to the bottom of articles that are pre-1950 or so. My guess is that there are a fair number of journals that practiced open peer review in the past that this article fails to mention and review. Understanding why open peer review was used and disappeared could provide more insight than observing journals, like PeerJ and F1000Research, that have only been trying this for two years.

I'd have to dig around for more examples but this article is one that has a "Communications" section appended to the end:

Wilson-Jones, R. A.: Steering and Stability of Single-Track Vehicles. Proceedings of the Institution of Mechanical Engineers: Automobile Division. 1951; 5(1): 191-199. Reference Source.

Competing Interests: No competing interests were disclosed. 
The benefits of publishing with F1000Research:

- Your article is published within days, with no editorial bias

- You can publish traditional articles, null/negative results, case reports, data notes and more

- The peer review process is transparent and collaborative

- Your article is indexed in PubMed after passing peer review

- Dedicated customer support at every stage

For pre-submission enquiries, contact research@f1000.com 